\title{
AN SU(3) APPROACH TO NUCLEAR MULTI-CLUSTER PROBLEMS †
}

\author{
K. T. HECHT and W. ZAHN \\ Department of Physics, The University of Michigan, Ann Arbor, Michigan, USA
}

Received 19 October 1978

\begin{abstract}
By combining SU(3) recoupling techniques with the use of the Bargmann-Segal integral transform closed expressions are derived for the interaction kernels of the nuclear cluster model. In a cluster model basis in which the relative motion and internal oscillator wave functions (with arbitrary and different oscillator frequencies) are expanded in SU(3) coupled form, SU(3) reduced matrix elements are evaluated by integral transform methods. Matrix elements in a basis of good angular momentum can be constructed for multi-cluster systems by combining these SU(3) reduced matrix elements with readily available $\mathrm{SU}(3)$ Wigner coefficients.
\end{abstract}

\section{Introduction}

The detailed treatment of nuclear cluster problems by resonating group methods has been made possible by many recent refinements in the resonating group technique. In particular, Hackenbroich's tremendous computer code has made possible sophisticated microscopic multichannel calculations ${ }^{1}$ ). Even though these computational techniques have been improved to allow extensive calculations in systems with mass as large as $A=8$, e.g. ref. ${ }^{2}$ ), detailed calculations of this type have nevertheless been limited to very light nuclei. The introduction of integral transform techniques $^{3-5}$ ) has furnished us with a powerful new tool for the evaluation of resonating group kernels by reducing the computational effort for nuclear cluster problems to a calculation of integrals over intercluster coordinates. Although much progress has been made in the technical problem of evaluating these integrals, some serious analytical difficulties remain. The evaluation of resonating group kernels in an angular momentum coupled basis has proved very difficult for cluster problems involving more than two fragments ${ }^{6}$ ) or for cluster problems with heavy fragments other than closed-shell nuclei such as ${ }^{16} \mathrm{O}$. A practical solution to both of these problems may be effected by exploiting the SU(3) symmetry properties of the relative motion and internal harmonic oscillator functions of the cluster basis, particularly if cluster functions are expressed in terms of an SU(3) coupled basis for which SU(3) recoupling techniques can be used to advantage ${ }^{7}$ ). In a basis in which the SU(3) quantum numbers of the several relative motion functions and internal functions of heavy

† Work supported by the National Science Foundation and the Deutsche Forschungsgemeinschaft, Bonn-Bad Godesberg, Germany. 
fragments are $\mathrm{SU}(3)$ coupled successively to resultants of good SU(3) symmetry, the details of the SU(3) subgroup labeling, including the angular momentum quantum. number, come into play only in the last step of the calculation. Difficulties associated with the fact that the angular momentum quantum number does not fit naturally into the SU(3) scheme are thus essentially avoided. In an SU(3) coupled cluster function basis SU(3) reduced matrix elements can be evaluated by integral transform techniques where any convenient basis (such as a Cartesian oscillator basis) can be used to effect the SU(3) coupling in the transform since the SU(3) reduced matrix elements are independent of SU(3) subgroup labels. Matrix elements in the physically meaningful basis of good angular momentum are then constructed trivially by combining the $S U(3)$ reduced matrix elements with readily available $S U(3) \supset R(3)$ Wigner coefficients $^{8}$ ). The technique can be applied to multi-cluster systems as readily as to simple cluster systems made up of only two fragments. The complexity of the cluster system is reflected only in the number of SU(3) recoupling coefficients needed for the evaluation of the reduced matrix elements.

Of the many integral transforms used in microscopic cluster calculations the Bargmann-Segal (BS) transform ${ }^{4}$ ) is ideally suited to the exploitation of SU(3) coupling techniques since oscillator functions have very simple SU(3) properties in Bargmann space. Many of the technical problems in setting up the transform of a resonating group kernel have been solved by earlier treatments. The problem of antisymmetrization can be handled by taking advantage of double coset formulations ${ }^{9}$ ). The coupling of the SU(4) symmetries of the various cluster fragments to build functions of good total SU(4) or conjugate space symmetry can be effected either by permutation group techniques in terms of a double coset formulation or by unitary group recoupling techniques ${ }^{9}$ ). The needed technology ${ }^{9}$ ) is readily available so that the problem has been reduced to one of evaluating orbital integrals. For the orbital problem the reduction to cluster relative coordinates has been carried through in a practical way in ref. ${ }^{4}$ ), and this will form the starting point of the present investigation. [The types of interactions to be considered are thus restricted to those of ref. ${ }^{10}$ ); two-body interactions are built from superposition of Gaussians.] Kernels of such interactions have transforms of simple Gaussian form for multicluster systems composed of fragments with 0 s internal functions ${ }^{5}$ ), hence each with mass $\leqq 4$. For cluster systems including heavier fragments the integral transforms of the resonating group kernels are products of Gaussians with polynomials in the transformed-space variables for the relative motion Jacobi vectors. The explicit construction of these polynomials has, however, been carried out in very few cases. The evaluation of such polynomials is relatively straightforward if they are constructed from SU(3) coupled tensors in the Bargmann-space variables for the relative motion vectors. In this form they can also be readily combined with the BS transform for the relative motion excitations by simple SU(3) coupling techniques. These lead directly to the SU(3) reduced matrix elements which are then combined with $\mathrm{SU}(3) \supset \mathrm{R}(3)$ Wigner coefficients to yield the matrix elements in the needed angular momentum basis. 
It is the purpose of this investigation to illustrate the new technique with a few very specific sample calculations. The illustration will proceed in two steps. In sect. 2 the simple three-cluster system, made up of three 0 s cluster fragments, will be treated by the new techniques. (For simplicity only central interactions are discussed to avoid the additional complications of spin couplings. These are extraneous to the SU(3) coupling techniques which are emphasized here but can easily be combined with them if spin-orbit and tensor forces are included.) A few numerical details are given for the ${ }^{12} \mathrm{C}=\alpha+\alpha+\alpha$ cluster system. There have been many studies of ${ }^{12} \mathrm{C}$. The purpose here is not to improve on these but to study the SU(3) irreducible tensor character of interactions which have been proved successful in earlier treatments of this cluster system ${ }^{11}$ ). In sect, 3 a fourth 0 s cluster fragment is added to show that the new technique can easily be generalized to the multicluster system. The $n$-cluster wave functions can also be made to include cluster systems with one or more heavy fragments by limiting the excitations of some of the relative motion functions in the $n$-cluster system to their lowest Pauli-allowed values. We shall indicate how the BS space polynomials generated by the $\mathrm{p}$-shell components of such a heavy fragment (with $5 \leqq A \leqq 16$ ) can be combined with the relative motion excitations of the remaining clusters to construct the full resonating group kernel for such a cluster system.

\section{The basic method and a simple example: The three-cluster system}

The three-cluster system used for illustration is described by a cluster wave function

$$
\Psi=\mathscr{A} \prod_{i=1}^{3} \varphi\left(\xi_{i}\right)^{(00)}\left[\chi\left(R_{1}\right)^{\left(Q_{1} 0\right)} \times \chi\left(R_{2}\right)^{\left(Q_{2} 0\right)}\right]_{x L M}^{(\lambda \mu)},
$$

where the internal wave functions, $\varphi$, of the three fragments are built from 0 s oscillator wave functions in the internal degrees of freedom $\xi_{i}$. The $\varphi$ include the full spinisospin function of the clusters. The relative motion functions, $\chi$, are harmonic oscillator functions in the Jacobi vectors $\boldsymbol{R}_{1}, \boldsymbol{R}_{2}$ which give the relative positions of the three cluster fragments. These carry $Q_{1}$ and $Q_{2}$ oscillator quanta and are characterized by their $\mathrm{SU}(3)$ labels $\left(\lambda_{i} \mu_{i}\right)=\left(Q_{i} 0\right)$, in Elliott's notation $\left.{ }^{12}\right)$. The function is given in $\mathrm{SU}(3)$ coupled form. The square bracket denotes $\mathrm{SU}(3)$ coupling. The resultant $\mathrm{SU}(3)$ symmetry $(\lambda \mu)$ is characterized by subgroup labels which include the angular momentum quantum numbers $L M$. To carry out the SU(3) coupling each $\chi(R)$ must be a harmonic oscillator function in a dimensionless variable $R$. Each dimensionless $R_{i}$ is equal to the physical $R_{i}$ (measured in $\mathrm{cm}$ or $\mathrm{fm}$ ) divided by the oscillator length parameter $\left[\hbar / M_{i} \omega_{i}\right]^{\frac{1}{2}}$ with appropriate reduced mass $M_{i}$ and frequency $\omega_{i}$ of whatever magnitude proves convenient. The method to be outlined will thus apply with equal validity whether different relative motion functions have equal or different oscillator frequencies. The antisymmetrizer, $\mathscr{A}$, handles the 
antisymmetrization between clusters. The matrix elements of an operator, such as the Hamiltonian, between such cluster functions can be converted to a kernel, $\mathscr{K}$, by performing the integrations over the internal variables $\xi$ for the three clusters. In our example the kernel will be a function of the variables $\bar{R}=\bar{R}_{1} \bar{R}_{2}, R=R_{1} R_{2}$ where the barred and unbarred quantities arise from the bra and ket of the full matrix element, respectively. Specifically, we want to develop a general technique for the evaluation of integrals of the type

$$
\begin{aligned}
I=\int \mathrm{d} \bar{R}_{1,2} \mathrm{~d} R_{1,2}\left[\chi^{*}\left(\bar{R}_{1}\right)^{\left(\bar{Q}_{1} 0\right)} \times \chi^{*}\left(\bar{R}_{2}\right)^{\left(\boldsymbol{Q}_{2} 0\right)}\right]_{\overline{\kappa L M}}^{\left(\bar{X}_{\mu}\right)} \\
\quad \times \mathscr{X}\left(\bar{R}_{1} R_{2} R_{1} R_{2}\right)\left[\chi\left(R_{1}\right)^{\left(Q_{1} 0\right)} \times \chi\left(R_{2}\right)^{\left(Q_{2} 0\right)}\right]_{\kappa L M,}^{(\lambda \mu)},
\end{aligned}
$$

between states of good total angular momentum $L$. (The kernel $\mathscr{K}$ is rotationally invariant; an $L=0$ operator.) If matrix elements are needed in an $L_{1} L_{2} L M$ [rather than the $(\lambda \mu) \kappa L M]$ basis it is only necessary to uncouple the $\mathrm{SU}(3)$ coupled relative motion functions:

$$
\begin{aligned}
& {\left[\chi\left(\boldsymbol{R}_{1}\right)^{\left(Q_{1} 0\right)} \times \chi\left(R_{2}\right)^{\left(Q_{2} 0\right)}\right]_{\kappa L M}^{(\lambda \mu)}} \\
& \quad=\sum_{L_{1} L_{2}}\left\langle\left(Q_{1} 0\right) L_{1} ;\left(Q_{2} 0\right) L_{2} \|(\lambda \mu) \kappa L\right\rangle\left(\chi_{L_{1}}\left(R_{1}\right)^{\left(Q_{1} 0\right)} \times \chi_{L_{2}}\left(R_{2}\right)^{\left(Q_{2} 0\right)}\right)_{L M},
\end{aligned}
$$

where the double-barred coefficient is an $\mathrm{SU}(3) \supset \mathrm{R}(3)$ reduced Wigner coefficient and the round bracket on the right-hand side now denotes only ordinary angular momentum coupling. The $\mathrm{SU}(3) \supset \mathbf{R}(3)$ Wigner coefficients are readily available through the computer code of Akiyama and Draayer ${ }^{8}$ ) (we use their conventions for the additional quantum number $\kappa$ ). If the kernal $\mathscr{K}$ is now imagined to be expanded in terms of SU(3) irreducible tensor components, $\mathscr{K}^{\left(\lambda_{0} \mu_{0}\right)}$, the integrals $I$ can be expanded in terms of $\mathrm{SU}(3)$ reduced matrix elements

$$
\begin{aligned}
I=\sum_{\left(\lambda_{0} \mu_{0}\right) \rho_{0}}\left\langle\bar{Q}_{1} \bar{Q}_{2}(\overline{\lambda \mu})\left\|\mathscr{X}^{\left(\lambda_{0} \mu_{0}\right)}\right\| Q_{1} Q_{2}(\lambda \mu)\right\rangle_{\rho_{0}} \\
\\
\times\left\langle(\bar{\lambda} \bar{\mu}) \bar{\kappa} L ;(\mu \lambda) \kappa L \|\left(\lambda_{0} \mu_{0}\right) L_{0}=0\right\rangle_{\rho_{0}}[2 L+1]^{-\frac{1}{2}} .
\end{aligned}
$$

The double barred (SU(3) reduced) matrix elements of $\mathscr{X}^{\left(\lambda_{0} \mu_{0}\right)}$ appear in combination with $\mathrm{SU}(3) \supset \mathbf{R}(3)$ reduced Wigner coefficients which carry the $L$-dependence. The sum includes a sum over the outer multiplicity label $\rho_{0}$ which is needed whenever the representation $\left(\lambda_{0} \mu_{0}\right)$ occurs with a $d$-fold multiplicity, with $d>1$ in the coupling $(\bar{\lambda} \bar{\mu}) \times(\mu \lambda)$. [The conventions of ref. ${ }^{8}$ ) are to be used for the multiplicity labels $\rho_{0}$. It is to be noted that the SU(3) reduced matrix elements are defined in terms of an unconventional order for the SU(3) coupling in the SU(3) Wigner coefficients. The more conventional order would have involved $\mathrm{SU}(3)$ Wigner coefficients $\langle(\lambda \mu) \kappa L$; $\left(\lambda_{0} \mu_{0}\right) 0 \|(\overline{\lambda \mu} \bar{\kappa} L\rangle_{\rho_{0}}$. In principle, the two coefficients are relatedby symmetry properties. In practice, these may not be simple in cases with outer multiplicity $\rho_{0}$ for $d>1$. The factor $[2 L+1]^{-1}=(-)^{L-M}\langle L M L-M \mid 00\rangle$ reflects the use of this 
symmetry for the ordinary angular momentum factor in the coupling.] It is to be emphasized that no explicit irreducible tensor decompositions of the kernel need to be carried out. The SU(3) reduced matrix elements of the kernel will fall out of the Bargmann-Segal (BS) transform of the kernel quite naturally if all factors in this transform are expressed in SU(3) coupled form. The full integrals $I$ in a basis of good angular momentum can thus be constructed through eq. (4) with the use of SU(3) Wigner coefficients which are accessible through efficient computer $\operatorname{codes}^{8}$ ).

The BS transform of the kernel, $\mathscr{K}$, can be constructed in terms of the BS transform kernels, $A$,

$$
H(\bar{K}, \boldsymbol{K})=\int A\left(\bar{K}^{\prime}, \bar{R}\right) \mathscr{K}(\overline{\mathbf{R}}, \boldsymbol{R}) A^{*}(K, R) \mathrm{d} \overline{\mathrm{R}} \mathrm{R},
$$

where in this case $\bar{K}, K$ stand collectively for $\bar{K}_{1} \bar{K}_{2}, K_{1} K_{2}$, the Bargmann-space variables which in transformed space correspond to $\bar{R}_{1}, \bar{R}_{2}$ and $R_{1} R_{2}$ [cf. eqs. $(2.3)-(2.9)$ of ref. $\left.\left.{ }^{4}\right)\right]$. In our specific case

$$
A(\boldsymbol{K}, \boldsymbol{R})=A\left(\boldsymbol{K}_{1}, R_{1}\right) A\left(\boldsymbol{K}_{2}, R_{2}\right),
$$

with

$$
\begin{gathered}
A\left(K_{1} R_{1}\right)=\prod_{i=x, y, z} A\left(K_{1}, R_{1}\right) \\
A\left(K_{x}, X\right)=\pi^{-\frac{1}{4}} \exp \left\{-\frac{1}{2} X^{2}-\frac{1}{2} K_{x}^{2}+\sqrt{2} X K_{x}\right\},
\end{gathered}
$$

where the harmonic.oscillator function generating function property of $A$ is of prime importance in our applications:

$$
A\left(K_{x}, X\right)=\sum_{n=0}^{\infty} \Psi_{n_{x}}^{*}(X) \frac{K_{x}^{n_{x}}}{\sqrt{n_{x} !}} .
$$

Here $K_{x}^{n_{x}} / \sqrt{n_{x}}$ ! is the normalized one-dimensional oscillator function in Bargmann space. The fact that the oscillator vacuum is the simple number 1 leads to much of the simplicity of Bargmann space.

In a Cartesian oscillator basis the Bargmann-space function

$$
P(K)_{n_{x} n_{y} n_{x}}^{(O)}=\frac{K_{x}^{n_{x}}}{\sqrt{n_{x}} !} \frac{K_{y}^{n_{y}}}{\sqrt{ } n_{y} !} \frac{K_{z}^{n_{x}}}{\sqrt{n_{y} !}}
$$

has SU(3) irreducible character $(Q 0)$ with $Q=n_{x}+n_{y}+n_{z}$, whereas

$$
P\left(K^{*}\right)_{n_{x} n_{y} n_{z}}^{(00)}=\frac{K_{x}^{* n_{x}}}{\sqrt{n_{x} !}} \frac{K_{y}^{* n_{y}}}{\sqrt{n_{y} !}} \frac{K_{z}^{* n_{x}}}{\sqrt{n_{z} !}}
$$

has SU(3) irreducible character $(0 Q)$.

An SU(3) coupled Bargmann-space polynomial can be defined by

$$
\begin{aligned}
& {\left[P\left(K_{1}\right)^{\left(Q_{1} 0\right)} \times P\left(K_{2}\right)^{\left(Q_{2} 0\right)}\right]_{\alpha}^{(\lambda \mu)}} \\
& \quad=\sum_{\alpha_{1} \alpha_{2}}\left\langle\left(Q_{1} 0\right) \alpha_{1}\left(Q_{2} 0\right) \alpha_{2} \mid(\lambda \mu) \alpha\right\rangle P\left(K_{1}\right)_{\alpha_{1}}^{\left(Q_{1} 0\right)} P\left(K_{2}\right)_{\alpha_{2}}^{\left(Q_{2} 0\right)},
\end{aligned}
$$


where subgroup labels $\alpha$ in the $U(3)$ coupling coefficient can be chosen in any convenient fashion; e.g. $\alpha=n_{x} n_{y} n_{z}$ in a Cartesian oscillator basis, or $\alpha=\kappa L M$ in an angular momentum basis. With $K_{1}=K_{2}$ a renormalization factor is needed. The normalized function $P(\boldsymbol{K})^{(\boldsymbol{Q} 0)}$ can be obtained from

$$
\left[P(K)^{\left(Q_{1} 0\right)} \times P(K)^{\left(Q_{2} 0\right)}\right]_{\alpha}^{(\lambda \mu)}=P_{\alpha}^{(Q 0)}(K)\left[\frac{Q !}{Q_{1} ! Q_{2} !}\right]^{\frac{1}{*}} \delta_{\lambda, Q=Q_{1}+Q_{2}} \delta_{\mu 0^{0}}
$$

In the Cartesian basis the $\mathrm{U}(3)$ coupling coefficient for the stretched coupling is given trivially in terms of binomial coefficients,

$$
\begin{aligned}
\left\langle(n 0) n_{x} n_{y} n_{z}(m 0) m_{x} m_{y} m_{z}\right|(n+m, 0) & \left.n_{x}+m_{x}, n_{y}+m_{y} n_{z}+m_{z}\right\rangle \\
= & {\left[\left(\begin{array}{c}
n_{x}+m_{x} \\
n_{x}
\end{array}\right)\left(\begin{array}{c}
n_{y}+m_{y} \\
n_{y}
\end{array}\right)\left(\begin{array}{c}
n_{z}+m_{z} \\
n_{z}
\end{array}\right) /\left(\begin{array}{c}
n+m \\
n
\end{array}\right)\right]^{\frac{1}{2}} . }
\end{aligned}
$$

The expansion of $A(\boldsymbol{K}, \boldsymbol{R})$ in the BS transform of the kernel $\mathscr{K}$ can now be expressed in terms of polynomials of the type (11):

$$
\begin{aligned}
& A(\bar{K}, \bar{R})=\sum_{\substack{n_{1_{x}} \ldots n_{2_{x}} \\
\left(\overline{\boldsymbol{Q}}_{i}=n_{i_{x}}+n_{i_{y}}+n_{i_{z}}\right)}} \sum_{(\overline{\lambda \bar{\mu}) \bar{z}}} \chi_{n_{1_{x}}}^{*}\left(\bar{X}_{1}\right) \chi_{n_{n_{y}}}^{*}\left(\bar{Y}_{1}\right) \chi_{n_{1_{x}}}^{*}\left(Z_{1}\right) \chi_{n_{n_{x}}}^{*}\left(\bar{X}_{2}\right) \chi_{n_{2_{y}}}^{*}\left(\bar{Y}_{2}\right) \chi_{n_{2_{z}}}^{*}\left(Z_{2}\right) \\
& \times\left\langle\left(\bar{Q}_{1} 0\right) n_{1_{x}} n_{1_{y}} n_{1_{x}}\left(\bar{Q}_{2} 0\right) n_{2_{x}} n_{2_{y}} n_{2_{x}} \mid(\bar{\lambda} \mu) \bar{\alpha}\right\rangle\left[P\left(\mathbb{K}_{1}\right)^{\left(\bar{Q}_{1} 0\right)} \times P\left(\bar{K}_{2}\right)^{\left(\bar{Q}_{2} 0\right)}\right]_{\bar{\alpha}}^{\left(\bar{\lambda}_{\bar{\alpha}} \bar{\mu}\right)}
\end{aligned}
$$

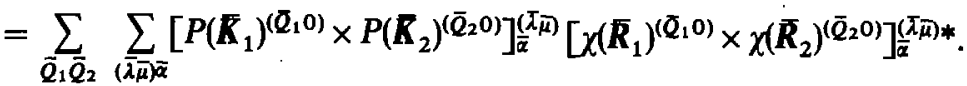

Since the sum over subgroup labels need not to be carried out explicitly, the U(3) Wigner coefficients can be supposed to be of the mixed variety where quantum numbers $\bar{\alpha}$ are given in the angular momentum basis $\bar{\alpha}=\bar{\kappa} L M$. With a similar expansion of $A^{*}(K, R)$ the BS transform of the kernel $\mathscr{K}$ can be expressed in the form.

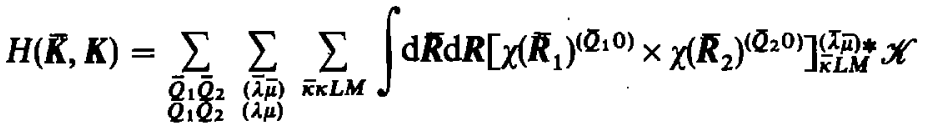

$$
\begin{aligned}
& \times\left[\chi\left(\boldsymbol{R}_{1}\right)^{\left(\boldsymbol{Q}_{1} 0\right)} \times \chi\left(\boldsymbol{R}_{2}\right)^{\left(Q_{2} 0\right)}\right]_{\kappa L M}^{(\lambda \mu)} \times\left[P\left(\overline{\boldsymbol{K}}_{1}\right)^{\left(\bar{Q}_{1} 0\right)} \times P\left(\bar{K}_{2}\right)^{\left(\bar{Q}_{2} 0\right)}\right]_{\bar{\kappa} L M}^{(\bar{\alpha} \mu)} \\
& \times\left[P\left(K_{1}^{*}\right)^{\left(0 Q_{1}\right)} \times P\left(K_{2}^{*}\right)^{\left(0 Q_{2}\right)}\right]_{\kappa L-M}^{(\mu \lambda)}(-)^{L-M} .
\end{aligned}
$$

By imagining an expansion of the kernel $\mathscr{K}$ in $\mathrm{SU}(3)$ irreducible tensors

$$
\mathscr{X}=\sum_{\left(\lambda_{0} \mu_{0}\right)} \mathscr{K}_{L_{0}=0}^{\left(\lambda_{0} \mu_{0}\right)}
$$

and expanding the integral through eq. (4) the sums over subgroup labels $\kappa \bar{\kappa} L M$ can be performed to express the BS transform of $\mathscr{K}^{\text {in }}$ terms of fully SU(3) coupled 
tensors in Bargmann space:

$$
\begin{aligned}
H(\overline{\boldsymbol{K}}, \boldsymbol{K}) & =\sum_{\substack{\left(\lambda_{0} \mu_{0}\right) \rho_{0} \\
\bar{Q}_{1} \bar{Q}_{2}}} \sum_{\substack{(\overline{\lambda \mu}) \\
Q_{1} Q_{2}}}\left\langle\bar{Q}_{1} \bar{Q}_{2}(\bar{\lambda} \bar{\mu})\left\|\mathscr{K}^{\left(\lambda_{0} \mu_{0}\right)}\right\| Q_{1} Q_{2}(\lambda \mu)\right\rangle_{\rho_{0}} \\
& \times\left[\left[P\left(\boldsymbol{K}_{1}\right)^{\left.\bar{Q}_{1} 0\right)} \times P\left(\boldsymbol{K}_{2}\right)^{\left(\overline{\boldsymbol{Q}}_{2} 0\right)}\right]^{(\bar{\lambda} \bar{\mu})} \times\left[P\left(\boldsymbol{K}_{1}^{*}\right)^{\left(0 Q_{1}\right)} \times P\left(\boldsymbol{K}_{2}^{*}\right)^{\left(0 Q_{2}\right)}\right]^{(\mu \lambda)}\right]_{L_{0}=0}^{\left(\lambda_{0} \mu_{0}\right) \rho_{0}} .
\end{aligned}
$$

The needed SU(3) reduced matrix elements of $\mathscr{K}$ can thus be read directly from the coefficients in an expansion of $H(\overline{\boldsymbol{K}}, \boldsymbol{K})$ in terms of the fully SU(3) coupled Bargmannspace tensors of eq. (17).

For the simple three-cluster system (or any multicluster system with fragments containing 0 s internal cluster wave functions) and interactions of the type used in refs. ${ }^{5,10}$, the BS transform $H(\bar{K}, \boldsymbol{K})$ is of simple Gaussian form

$$
H(\overline{\boldsymbol{K}}, \boldsymbol{K})=\sum_{\beta} a_{\beta} \exp \{\rho(\beta)\} \exp \{\sigma(\beta)\} \exp \{\tau(\beta)\} .
$$

The $\beta$-sum arises from the antisymmetrizer which is expressed in terms of a double coset formulation ${ }^{9}$ ). The $\beta$-sum runs over the full set of double coset generators; the weighting factors $a_{\beta}$ are calculated by the techniques of ref. ${ }^{9}$ ) (actual sums are performed by simple computer codes). The Gaussian functions $\exp \{\rho\}, \exp \{\sigma\}$, $\exp \{\tau\}$ are abbreviations for

$$
\begin{aligned}
& \exp \{\sigma(\beta)\} \equiv \exp \left\{\sum_{i, j=1}^{2} \sigma_{i j}(\beta)\left(\overline{\boldsymbol{K}}_{i} \cdot \boldsymbol{K}_{j}^{*}\right)\right\}, \\
& \exp \{\rho(\beta)\} \equiv \exp \left\{\sum_{i, j=1}^{2} \rho_{i j}(\beta)\left(\overline{\boldsymbol{K}}_{i} \cdot \overline{\boldsymbol{K}}_{j}\right)\right\}, \\
& \exp \{\tau(\beta)\} \equiv \exp \left\{\sum_{i, j=1}^{2} \tau_{i j}(\beta)\left(\boldsymbol{K}_{i}^{*} \cdot \boldsymbol{K}_{j}^{*}\right)\right\},
\end{aligned}
$$

where the matrix elements $\sigma_{i j}, \rho_{i j}, \tau_{i j}$ for each $\beta$ are calculated by the analytic methods of ref. ${ }^{5}$ ).

The exp $\{\sigma\}$ factor is an SU(3) scalar so that its expansion in terms of SU(3) coupled $\boldsymbol{K}$-space polynomials is particularly simple. The basic building block is expressed as

$$
\left(\overline{\boldsymbol{K}}_{i} \cdot \boldsymbol{K}_{j}^{*}\right)=\sqrt{3}\left[P\left(\overline{\boldsymbol{K}}_{i}\right)^{(10)} \times P\left(\boldsymbol{K}_{j}^{*}\right)^{(01)}\right]_{L=0}^{(00)}
$$

With the use of eq. (12) and the simple SU(3) recoupling coefficient

we obtain

$$
\left[\begin{array}{lll}
(n 0) & (0 n) & (00) \\
(m 0) & (0 m) & (00) \\
(\lambda \mu) & (\mu \lambda) & (00)
\end{array}\right]=\left[\begin{array}{c}
\operatorname{dim}(\lambda \mu) \\
\operatorname{dim}(n 0) \operatorname{dim}(m 0)
\end{array}\right]^{\frac{1}{2}},
$$

$$
\frac{\left(\overline{\boldsymbol{K}}_{i} \cdot \boldsymbol{K}_{j}^{*}\right)^{Q}}{Q !}=\left[P\left(\overline{\boldsymbol{K}}_{i}\right)^{(Q 0)} \times P\left(\boldsymbol{K}_{j}^{*}\right)^{(0 Q)}\right]_{0}^{(00)}[\operatorname{dim}(Q 0)]^{\frac{1}{2}}
$$


where $\operatorname{dim}(\lambda \mu)=\frac{1}{2}(\lambda+1)(\mu+1)(\lambda+\mu+2)$ is the dimension of $(\lambda \mu)$. The $9(\lambda \mu)$ coefficient in square brackets is a particularly simple SU(3) recoupling coefficient in unitary form (the arrangement of rows and columns follows the standard notation for the $9 j$ symbol of angular momentum recoupling theory). Straightforward $S U(3)$ recoupling [with the use of eqs. (12) and (21)] leads to the expansion of the $\exp \{\sigma\}$ factor

$$
\begin{aligned}
\exp \{\sigma\} & =\sum_{\left(\lambda_{\sigma} \mu_{\sigma}\right)} \sum_{\substack{l+m, n+j \\
l+m, m+j}} C_{\sigma}\left(l+m, n+j, l+n, m+j ;\left(\lambda_{\sigma} \mu_{\sigma}\right)\right) \\
\times & {\left[\left[P\left(\bar{K}_{1}\right)^{(l+m, 0)} \times P\left(\bar{K}_{2}\right)^{(n+j, 0)}\right]^{\left(\lambda_{\sigma} \mu_{\sigma}\right)} \times\left[P\left(K_{1}^{*}\right)^{(0, l+n)} \times P\left(K_{2}^{*}\right)^{(0, m+j)}\right]^{\left(\mu_{\sigma} \lambda_{\sigma}\right)}\right]_{0}^{(00)}, }
\end{aligned}
$$

with

$$
\begin{aligned}
C_{\sigma}= & \sum_{\substack{l \\
(m, n, j)}} \sigma_{11}^{l} \sigma_{12}^{m} \sigma_{21}^{n} \sigma_{22}^{j}\left[\operatorname{dim}\left(\lambda_{\sigma} \mu_{\sigma}\right)\right]^{\frac{1}{2}} \\
& \times\left[\left(\begin{array}{c}
l+m \\
l
\end{array}\right)\left(\begin{array}{c}
n+j \\
j
\end{array}\right)\left(\begin{array}{c}
l+n \\
l
\end{array}\right)\left(\begin{array}{c}
m+j \\
j
\end{array}\right)\right]^{\frac{1}{t}}\left(\begin{array}{ccc}
(l 0) & (m 0) & (l+m, 0) \\
(n 0) & (j 0) & (n+j, 0) \\
(l+n, 0) & (m+j, 0) & \left(\lambda_{\sigma} \mu_{\sigma}\right)
\end{array}\right),
\end{aligned}
$$

where the $\mathrm{U}(3) 9(\lambda \mu)$ coefficient contains at most two-rowed representations so that it is equivalent to an $S U(2) X$-coefficient (unitary form of the $9 j$ recoupling coefficient). With the "stretched" coupling it can moreover be related to an SU(3) Wigner coefficient [cf., e.g. eq. (3.8) of ref. $\left.{ }^{13}\right)$ ].

The basic building block of the $\exp \{\rho\}$ factor is an SU(3) irreducible (20) tensor with $L=0$.

With $i \neq j$ :

$$
\left(\boldsymbol{K}_{i} \cdot \boldsymbol{K}_{j}\right)=\sqrt{3}\left[P\left(\boldsymbol{K}_{i}\right)^{(10)} \times P\left(\overline{\boldsymbol{K}}_{j}\right)^{(10)}\right]_{L=0}^{(20)}
$$

and with $i=j$ :

$$
\left.\left(\bar{K}_{i} \cdot \bar{K}_{i}\right)=\sqrt{6 P\left(\bar{K}_{i}\right.}\right)_{L=0}^{(20)} .
$$

Using algebraic expressions ${ }^{14}$ ) for the simple $\mathrm{SU}(3) \supset \mathbf{R}(3)$ Wigner coefficients $\left\langle\left(Q_{1} 0\right) 0 ;\left(Q_{2} 0\right) 0 \|(\lambda \mu) 0\right\rangle$ and very simple $9(\lambda \mu)$ recoupling coefficients it is easy to show that with $i \neq j$ :

$$
\frac{\left(\overline{\boldsymbol{K}}_{i} \cdot \overline{\boldsymbol{K}}_{j}\right)^{Q}}{Q !}=\sum_{(\lambda \mu)}(\lambda+1)^{ \pm}(-)^{\frac{1}{2} \mu}\left[P\left(\bar{K}_{i}\right)^{(Q 0)} \times P\left(\bar{K}_{j}\right)^{(Q 0)}\right]_{L=0}^{(\lambda \mu)}
$$

where $(\lambda \mu)$ runs over the values $(2 Q, 0),(2 Q-4,2),(2 Q-8,4), \ldots,(0, Q)$ for $Q=$ even or $\ldots,(2, Q-1)$ for $Q=$ odd, and with $i=j$ :

$$
\frac{\left(\boldsymbol{K}_{i} \cdot \overline{\boldsymbol{K}}_{i}\right)^{Q}}{Q !}=\frac{[(2 Q+1) !]^{\frac{1}{2}}}{Q !} P\left(\bar{K}_{i}\right)_{L=0}^{(2 Q .0)}
$$


[Since the phases are partly a question of convention it should be pointed out that the phases of eq. (26) are in agreement with the phase convention of Akiyama and Draayer ${ }^{8}$ ) if $\mathrm{SU}(3) / \mathrm{R}(3)$ states are defined in terms of the labels $I J=01$ of ref. ${ }^{8}$ ) for all $(\lambda \mu)$.]

The expansion of the $\exp \{\rho\}$ factor in terms of $\mathrm{SU}(3)$ irreducible tensors,

$$
\exp \{\rho\}=\sum_{\bar{q}, \bar{q}_{2}} \sum_{\left(\lambda_{\rho} \mu_{\rho}\right)} B_{\rho}\left(\bar{q}_{1}, \bar{q}_{2} ;\left(\lambda_{\rho} \mu_{\rho}\right)\right)\left[P\left(\bar{K}_{1}\right)^{\left(\bar{q}_{1} 0\right)} \times P\left(\bar{K}_{2}\right)^{\left(\overline{\bar{q}}_{2} 0\right)}\right]_{\mathbf{L}_{\rho}=0}^{\left(\lambda_{\rho} \mu_{\rho}\right)}
$$

is best carried out by introducing an intermediate unitary (orthogonal) transformation

$$
\begin{aligned}
& \overline{\boldsymbol{K}}_{a}=\cos \left(\frac{1}{2} \beta\right) \overline{\boldsymbol{K}}_{1}-\sin \left(\frac{1}{2} \beta\right) \overline{\boldsymbol{K}}_{2}, \\
& \overline{\boldsymbol{K}}_{b}=\sin \left(\frac{1}{2} \beta\right) \overline{\boldsymbol{K}}_{1}+\cos \left(\frac{1}{2} \beta\right) \boldsymbol{K}_{2},
\end{aligned}
$$

where $\beta$ is chosen so that

$$
\exp \{\rho\}=\exp \left\{\rho_{a a}\left(\overline{\boldsymbol{K}}_{a} \cdot \overline{\boldsymbol{K}}_{a}\right)+\rho_{b b}\left(\overline{\boldsymbol{K}}_{b} \cdot \overline{\boldsymbol{K}}_{b}\right)\right\}
$$

with

$$
\begin{gathered}
\rho_{a a}=\rho_{11} \cos ^{2} \frac{1}{2} \beta+\rho_{22} \sin ^{2} \frac{1}{2} \beta-2 \rho_{12} \cos \frac{1}{2} \beta \sin \frac{1}{2} \beta, \\
\rho_{b b}=\rho_{11} \sin ^{2} \frac{1}{2} \beta+\rho_{22} \cos ^{2} \frac{1}{2} \beta+2 \rho_{12} \cos \frac{1}{2} \beta \sin \frac{1}{2} \beta, \\
\tan \beta=\frac{2 \rho_{12}}{\rho_{22}-\rho_{11}} .
\end{gathered}
$$

The transformation from the $\overline{\boldsymbol{K}}_{a}, \overline{\boldsymbol{K}}_{b}$ to the $\boldsymbol{K}_{1}, \overline{\mathbf{K}}_{2}$ basis is then made by the unitary transformation

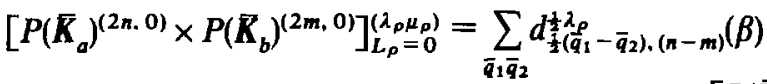

$$
\begin{aligned}
& \times\left[P\left(\bar{K}_{1}\right)^{\left(\bar{q}_{1} 0\right)} \times P\left(\bar{R}_{2}\right)^{\left(\bar{q}_{2} 0\right)}\right]_{L_{\rho}=0}^{\left(\lambda_{\rho} \mu_{\rho}\right)},
\end{aligned}
$$

where the $S U(2) d$-function [using the phase convention of eq. (4.1.15) of ref. $\left.{ }^{15}\right)$ ] is the generalized Moshinsky bracket for SU(3) coupled oscillator functions, [as shown by Brody and Moshinsky ${ }^{16}$ ) and Kaufman and Noack $\left.{ }^{17}\right)$ ]. The expansion of eq. (29), with the use of eqs. (26b) and (32), leads to the expansion coefficients

$$
\begin{aligned}
& B_{\rho}\left(\bar{q}_{1} \bar{q}_{2} ;\left(\lambda_{\rho} \mu_{\rho}\right)\right)=\sum_{\substack{n, m \\
n+m=t\left(\bar{q}_{1}+\bar{q}_{2}\right)}} \rho_{a \rho}^{n} \rho_{b b}^{m} \\
& \times \frac{[(2 n+1) !(2 m+1) !]^{\frac{1}{2}}}{n ! m !}\left\langle(2 n, 0) 0 ;(2 m, 0) 0 \|\left(\lambda_{\rho} \mu_{\rho}\right) 0\right\rangle \times d_{\frac{1}{2}\left(\hat{q}_{1}-\bar{q}_{2}\right),(n-m)}^{\lambda_{2}}(\beta) .
\end{aligned}
$$

The basic building block of the $\exp \{\tau\}$ factor is an SU(3) irreducible tensor of rank (02):

$$
\left(K_{i}^{*} \cdot K_{i}^{*}\right)=\sqrt{6} P\left(K_{i}^{*}\right)_{L=0}^{(02)} ; \quad\left(K_{i}^{*} \cdot K_{j}^{*}\right)=\sqrt{3}\left[P\left(K_{i}^{*}\right)^{(01)} \times P\left(K_{j}^{*}\right)^{(01)}\right]_{L=0}^{(02)} .
$$


Simple conjugation properties of the SU(3) tensors show that the expansion of the $\exp \{\tau\}$ factor is given by $B$-coefficients of the type of eq. (33).

Finally, the Bargmann-space SU(3) tensors from the expansions of the $\exp \{\rho\}$, $\exp \{\sigma\}$ and $\exp \{\tau\}$ factors are $\mathrm{SU}(3)$ coupled successively to resultant $\left(\lambda_{0} \mu_{0}\right)$, in a $\left[\left[\left(\lambda_{\rho} \mu_{\rho}\right) \times(00)\right]\left(\lambda_{\rho} \mu_{\rho}\right) \times\left(\mu_{\tau} \lambda_{\tau}\right)\right]\left(\lambda_{0} \mu_{0}\right) \rho_{0}$ scheme, where the SU(3) coupled tensors of the form

$$
\begin{aligned}
{\left[\left[\left[P\left(\boldsymbol{K}_{1}\right)^{\left(\bar{q}_{1} 0\right)} \times P\left(\mathbb{K}_{2}\right)^{\left(\bar{q}_{2} 0\right)}\right]^{\left(\lambda_{\rho} \mu_{\rho}\right)}\right.\right.} \\
\times\left[\left[P\left(\bar{K}_{1}\right)^{\left(\overline{\boldsymbol{Q}}_{1}-\bar{q}_{1}, 0\right)} \times P\left(\bar{K}_{2}\right)^{\left(\overline{\boldsymbol{Q}}_{2}-\bar{q}_{2}, 0\right)}\right]^{\left(\lambda_{\sigma} \mu_{\sigma}\right)}\right. \\
\left.\left.\times\left[P\left(\boldsymbol{K}_{1}^{*}\right)^{\left(0, Q_{1}-q_{1}\right)} \times P\left(\boldsymbol{K}_{2}^{*}\right)^{\left(0, Q_{2}-q_{2}\right)}\right]^{\left(\mu_{\sigma} \lambda_{\sigma}\right)}\right]^{(00)}\right]^{\left(\lambda_{\rho} \mu_{\rho}\right)} \\
\left.\times\left[P\left(\boldsymbol{K}_{1}^{*}\right)^{\left(0 q_{1}\right)} \times P\left(\boldsymbol{K}_{2}^{*}\right)^{\left(0 q_{2}\right)}\right]^{\left(\mu_{\tau} \lambda_{\tau}\right)}\right]_{L_{0}=0}^{\left(\lambda_{0} \mu_{0}\right) \rho_{0}}
\end{aligned}
$$

are then transformed into the needed

$$
\left[\left[P\left(\bar{K}_{1}\right)^{\left(\overline{\boldsymbol{Q}}_{1} 0\right)} \times P\left(\bar{K}_{2}\right)^{\left(\bar{Q}_{2} 0\right)}\right]^{(\bar{\lambda} \bar{\mu})} \times\left[P\left(\bar{K}_{1}^{*}\right)^{\left(0 Q_{1}\right)} \times P\left(K_{2}^{*}\right)^{\left(0 Q_{2}\right)}\right]^{(\mu \lambda)}\right]_{L_{0}=0}^{\left(\lambda_{0} \mu_{0}\right) \rho_{0}}
$$

form by straightforward SU(3) recoupling to yield

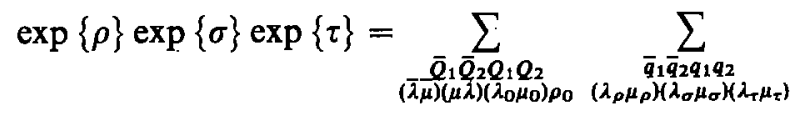

$$
\begin{aligned}
& \times C_{\sigma}\left(\bar{Q}_{1}-\bar{q}_{1}, \bar{Q}_{2}-\bar{q}_{2}, Q_{1}-q_{1}, Q_{2}-q_{2} ;\left(\lambda_{\sigma} \mu_{\sigma}\right)\right) \\
& \times B_{\rho}\left(\bar{q}_{1}, \bar{q}_{2},\left(\lambda_{\rho} \mu_{\rho}\right)\right) B_{\tau}\left(q_{1} q_{2},\left(\lambda_{\tau} \mu_{\tau}\right)\right) \\
& \times Z\left(\bar{Q}_{1} \bar{Q}_{2} Q_{1} Q_{2} \bar{q}_{1} \bar{q}_{2} q_{1} q_{2}\left(\lambda_{\rho} \mu_{\rho}\right)\left(\lambda_{\sigma} \mu_{\sigma}\right)\left(\lambda_{\tau} \mu_{\tau}\right)(\bar{\lambda} \mu)(\lambda \mu)\left(\lambda_{0} \mu_{0}\right) \rho_{0}\right) \\
& \times\left[\left[P\left(\overline{\boldsymbol{K}}_{1}\right)^{\left(\overline{\boldsymbol{Q}} \overline{1}^{0}\right)} \times P\left(\bar{K}_{2}\right)^{\left(\overline{\boldsymbol{Q}}_{2} 0\right)}\right]^{(\overline{\bar{\lambda}} \bar{\mu})}\right. \\
& \left.\times\left[P\left(K_{1}^{*}\right)^{\left(0 Q_{1}\right)} \times P\left(K_{2}^{*}\right)^{\left(0 Q_{2}\right)}\right]^{(\mu \lambda)}\right]_{L_{0}=0}^{\left(\lambda_{0} \mu_{0}\right) \rho_{0}},
\end{aligned}
$$

where

$$
\begin{aligned}
Z= & {\left[\frac{\operatorname{dim}(\overline{\lambda \mu})}{\operatorname{dim}\left(\lambda_{\rho} \mu_{\rho}\right) \operatorname{dim}\left(\lambda_{\sigma} \mu_{\sigma}\right)}\right]^{\frac{1}{2}}(-)^{\lambda_{\rho}+\mu_{\rho}+\lambda_{\sigma}+\mu_{\sigma}-\bar{\lambda}-\bar{\mu}} } \\
& \times\left\{\sum _ { \overline { \rho } _ { 0 } } \left\langle\left(\lambda_{\rho} \mu_{\rho}\right) L_{\rho}=0 ;\left(\mu_{\tau} \lambda_{\tau}\right) L_{\tau}=0 \|\left(\left(\lambda_{0} \mu_{0}\right) L_{0}=0\right\rangle_{\bar{\rho}_{0}}\right.\right. \\
& \left.\times U\left((\overline{\lambda \mu})\left(\mu_{\sigma} \lambda_{\sigma}\right)\left(\lambda_{0} \mu_{0}\right)\left(\dot{\mu}_{\tau} \lambda_{\tau}\right) ;\left(\lambda_{\rho} \mu_{\rho}\right) 1 \bar{\rho}_{0} ;(\mu \lambda) 1 \rho_{0}\right)\right\}
\end{aligned}
$$




$$
\begin{aligned}
\times\left[\left(\begin{array}{c}
\bar{Q}_{1} \\
\bar{q}_{1}
\end{array}\right)\left(\begin{array}{l}
\bar{Q}_{2} \\
\bar{q}_{2}
\end{array}\right)\left(\begin{array}{l}
Q_{1} \\
q_{1}
\end{array}\right)\left(\begin{array}{l}
Q_{2} \\
q_{2}
\end{array}\right)\right]^{\frac{1}{2}} \\
\\
{\left[\begin{array}{ccc}
\left(\bar{q}_{1} 0\right) & \left(\bar{q}_{2} 0\right) & \left(\lambda_{\rho} \mu_{\rho}\right) \\
\left(\bar{Q}_{1}-\bar{q}_{1}, 0\right) & \left(\bar{Q}_{2}-\bar{q}_{2}, 0\right) & \left(\hat{\lambda}_{\sigma} \mu_{\sigma}\right) \\
\left(\bar{Q}_{1} 0\right) & \left(\bar{Q}_{2} 0\right) & (\overline{\lambda \mu})
\end{array}\right]\left[\begin{array}{ccc}
\left(Q_{1}-q_{1}, 0\right) & \left(Q_{2}-q_{2}, 0\right) & \left(\hat{\lambda}_{\sigma} \mu_{\sigma}\right) \\
\left(q_{1} 0\right) & \left(q_{2} 0\right) & \left(\lambda_{\tau} \mu_{\tau}\right) \\
\left(Q_{1} 0\right) & \left(Q_{2} 0\right) & (\lambda \mu)
\end{array}\right] .(3) }
\end{aligned}
$$

The SU(3) reduced matrix elements which are the coefficients of the Bargmannspace tensors of the type of eq. (36) [see also eq. (17)] can thus be evaluated in terms of the coefficients $C_{\sigma}, B_{\rho}, B_{\tau}$ and the coefficient $Z$ which carries the SU(3) recoupling coefficients. The $U$-coefficient is an SU(3) Racah coefficient in unitary form and is written in the notation of ref. ${ }^{8}$ )

$$
U\left(\left(\lambda_{1} \mu_{1}\right)\left(\lambda_{2} \mu_{2}\right)(\lambda \mu)\left(\lambda_{3} \mu_{3}\right) ;\left(\lambda_{12} \mu_{12}\right) \rho_{12} \rho_{12,3} ;\left(\lambda_{23} \mu_{23}\right) \rho_{23} \rho_{1,23}\right),
$$

which is a direct generalization from ordinary angular momentum theory. These $U$-coefficients are readily available through the code of Akiyama and Draayer ${ }^{8}$ ). The two $9(\lambda \mu)$ recoupling coefficients contain at most two-rowed $\mathrm{U}(3)$ representations so that they are equivalent to $\mathrm{SU}(2) X$-coefficients (unitary form of the $9 j$ recoupling coefficients). With two "stretched" couplings these again have a very simple form. Since $\left(\lambda_{\rho} \mu_{\rho}\right)\left(\lambda_{\sigma} \mu_{\sigma}\right)$ and $\left(\lambda_{\tau} \mu_{\tau}\right)$ all correspond to two-rowed U(3) representations $\left(\bar{\lambda}+2 \bar{\mu}=\lambda_{\rho}+2 \mu_{\rho}+\lambda_{\sigma}+2 \mu_{\sigma}\right)$, the coupling $(\overline{\lambda \mu}) \times\left(\mu_{\sigma} \lambda_{\sigma}\right) \rightarrow\left(\lambda_{\rho} \mu_{\rho}\right)$ is free of multiplicity; the label $\rho$ in the 12 position of the $U$-coefficient can thus only have the value 1 and is essentially redundant. Similar remarks hold for the $\left(\mu_{\sigma} \lambda_{\sigma}\right) \times\left(\mu_{\tau} \lambda_{\tau}\right) \rightarrow(\mu \lambda)$ coupling and the label $\rho$ in the 23 position.

To illustrate the complexity of the $\vec{q}_{i}, q_{i},\left(\lambda_{\rho} \mu_{\rho}\right),\left(\lambda_{\sigma} \mu_{\sigma}\right),\left(\lambda_{\tau} \mu_{\tau}\right)$ structure in a specific case, it may be useful to consider a very simple example, the ${ }^{12} \mathrm{C}=\alpha+\alpha+\alpha$ cluster system with oscillator functions of equal frequency. For this system the lowest Pauli-allowed states have $\bar{Q}_{1}=\bar{Q}_{2}=Q_{1}=Q_{2}=4$ and $(\overline{\lambda \mu})=(\lambda \mu)=(04)$. The possible values of $\left(\lambda_{\rho} \mu_{\rho}\right),\left(\lambda_{\sigma} \mu_{\sigma}\right),\left(\lambda_{\tau} \mu_{t}\right)$ for this subspace of the cluster basis are listed in table 1 . In this subspace the SU(3) tensor components $\mathscr{K}^{\left(\lambda_{0} \mu_{0}\right)}$ are restricted to the possibilities $\left(\lambda_{0} \mu_{0}\right)=(00),(22),(44)$. The relative importance of the three $\left(\lambda_{0} \mu_{0}\right)$ tensors is determined for the contributions from each $\bar{q}_{1}+\bar{q}_{2}=q_{1}+q_{2}$ term by the sum in the curly brackets of eq. (37b). These quantities are therefore included in table 1.

A realistic calculation will clearly require either a much larger pure oscillator basis or, preferably, will stem from a variational treatment in which radial functions are expanded in terms of oscillator functions of several different frequencies. Insofar as all of our techniques make use of oscillator functions $\chi(R)$ in dimensionless variables $\boldsymbol{R}$, with unit weighting factors in the BS transform kernels $A(\boldsymbol{K}, \boldsymbol{R})$, they apply with equal validity to the case where different relative motion functions have different frequencies, and frequencies different from the 0 s internal cluster functions. The different $\omega_{i}$ merely lead to a more complicated set of $\rho_{i j}, \sigma_{i j}, \tau_{i j}$, with more complicated 
TABLE 1

Composition of the $\left(\lambda_{0} \mu_{0}\right)$ structure for the lowest Pauli-allowed states of ${ }^{12} \mathrm{C}$

\begin{tabular}{cccccc}
\hline$\left.\left(q_{1}+q_{2}\right)^{\prime \prime}\right)$ & $\left(\lambda_{0} \mu_{0}\right)$ & $\left(\lambda_{\sigma} \mu_{\sigma}\right)$ & $(\lambda \mu)$ & $\left(\hat{\lambda}_{\tau} \mu_{\tau}\right)$ & \{\}$\left.^{\mathrm{b}}\right)$ \\
\hline 0 & $(00)$ & $(04)$ & $(00)$ & $(00)$ & 1 \\
2 & $(00)$ & $(22)$ & $(20)$ & $(20)$ & $\sqrt{\frac{1}{6}}$ \\
2 & $(22)$ & $(22)$ & $(20)$ & $(20)$ & $-\frac{1}{9} \sqrt{\frac{7}{3}}$ \\
4 & $(00)$ & $(40)$ & $(40)$ & $(40)$ & $\sqrt{\frac{1}{15}}$ \\
4 & $(22)$ & $(40)$ & $(40)$ & $(40)$ & $-\frac{1}{3} \sqrt{\frac{7}{30}}$ \\
4 & $(44)$ & $(40)$ & $(40)$ & $(40)$ & $\frac{1}{5} \sqrt{\frac{7}{10}}$ \\
4 & $(00)$ & $(02)$ & $(02)$ & $(02)$ & $\sqrt{\frac{1}{6}}$ \\
4 & $(22)$ & $(02)$ & $(02)$ & $(02)$ & $-\frac{1}{3} \sqrt{\frac{7}{3}}$ \\
6 & $(00)$ & $(20)$ & $(22)$ & $(22)$ & $\frac{1}{3} \sqrt{\frac{1}{3}}$ \\
6 & $(22)$ & $(20)$ & $(22)$ & $(22)$ & $-\frac{2}{15} \sqrt{\frac{14}{3}}$ \\
6 & $(44)$ & $(20)$ & $(22)$ & $(22)$ & $\frac{1}{15} \sqrt{14}$ \\
8 & $(00)$ & $(00)$ & $(04)$ & $(04)$ & $\sqrt{\frac{1}{15}}$ \\
8 & $(22)$ & $(00)$ & $(04)$ & $(04)$ & $-\sqrt{\frac{7}{30}}$ \\
8 & $(44)$ & $(00)$ & $(04)$ & $(04)$ & $\sqrt{\frac{7}{10}}$ \\
\hline
\end{tabular}

2) Note that $\bar{q}_{1}+\bar{q}_{2}=q_{1}+q_{2}$.

b) \{ \} stands for the sum in the curly bracket of eq. (37b).

weighting coefficients ${ }^{4}$ ). In addition, the distinction between Pauli-allowed and Pauli-forbidden $Q_{i}$ values disappears when the different relative motion functions have different frequencies. Even very low values of $Q_{1}$ and $Q_{2}$ should thus be included in the basis in variational treatments with radial functions expanded in terms of oscillator functions of several different frequencies. Our aim here is not to improve on earlier calculations for ${ }^{12} \mathrm{C}$ but to study the SU(3) irreducible tensor character of interactions of the type $\left.{ }^{10}\right)$ used in earlier successful treatments of the $(\alpha+\alpha+\alpha)$ cluster system. For this reason only matrix elements in the $\alpha+\alpha+\alpha$ cluster basis with $Q_{1}=Q_{2}=4,(\lambda \mu)=(04)$ will be considered. However, these will be derived from a set of $\rho_{i j}, \sigma_{i j}$, and $\tau_{i j}$ which stem from a realistic variational trial function for ${ }^{12} \mathrm{C}$ which can be expected to give both reasonable values for the binding energy and the excitations of the $0^{+}, 2^{+}, 4^{+}$rotational band of ${ }^{12} \mathrm{C}$.

The Hamiltonian, successfully employed by the Hackenbroich group in Cologne for mass numbers $3 \leqq A \leqq 12$ is built from a kinetic energy term and a nucleonnucleon soft-core model potential ${ }^{10}$ ) with central and Coulomb components. The spin-orbit and tensor components of this potential are not needed in the ${ }^{12} \mathrm{C}=\alpha+\alpha+\alpha$ system. The central and Coulomb potentials are made up from superpositions of Gaussians ${ }^{10}$ ). This Hamiltonian requires the use of Jastrow correlations

$$
f=\prod_{k<l=1}^{12}\left(1-d_{1} \exp \left\{-d_{2}\left(r_{k}-r_{l}\right)^{2}\right\}\right)
$$

with $d_{1}=0.6$ and $d_{2}=3.0 \mathrm{fm}^{-2}$. The short-range correlations are shifted by an 
approximate treatment from the wave functions to the Hamiltonian $f H f \rightarrow H_{\text {eff }}$ This procedure is made necessary by limited computer capacities and has been described in detail in ref. ${ }^{1}$ ).

This Hamiltonian has been used in a basis in which the orbital parts of the $\alpha$ particle internal wave functions are given by

$$
\prod_{i=1}^{3} \exp \left\{-\frac{1}{4} a \sum_{i>j=1}^{4} r_{i j}^{2}\right\}
$$

with $a=0.27 \mathrm{fm}^{-2}$, yielding a binding energy of $-21.51 \mathrm{MeV}$ for the $\alpha$-particle. The exponential parts of the relative motion functions, $\chi$, are given in terms of Ritz variational parameters, $b_{i x}$, by

$$
\sum_{x=1}^{5} b_{i x} \exp \left\{\dot{-} \eta_{i x} R_{i}^{2}\right\}
$$

Each radial function is taken as a superposition of five Gaussians, with parameters $\eta_{11}=0.28, \eta_{12}=0.22, \eta_{13}=0.15, \eta_{14}=0.05, \eta_{15}=0.01$ in the first Jacobi coordinate, $\boldsymbol{R}_{1}$, and $\eta_{21}=0.34, \eta_{22}=0.20, \eta_{23}=0.12, \eta_{24}=0.03, \eta_{25}=0.005$ in the second Jacobi coordinate, $\boldsymbol{R}_{2}$. All values are given in $\mathrm{fm}^{-2}$. The superposition of wave functions with different oscillator frequencies in one and the same variable requires - almost trivial - dilation techniques as outlined in ref. ${ }^{4}$ ). With $b_{i \alpha}$ determined by minimizing the groud state energy for ${ }^{12} \mathrm{C}$, a set of $\rho_{i j}, \sigma_{i j}, \tau_{i j}$ has been calculated.

Even though a correspondingly large basis, starting with low values of $Q_{1}, Q_{2}$, should now be chosen for a complete calculation, the cluster states with $Q_{1}=Q_{2}=4$, $(\lambda \mu)=(04)$ have been singled out specifically to study the SU(3) irreducible tensor character of the above interaction. The magnitudes of the SU(3) reduced matrix elements for this interaction in the restricted $Q_{1}=Q_{2}=4,(\lambda \mu)=(04)$ cluster basis are shown in table 2 . It is interesting to note that the $\mathrm{SU}(3)$ scalar components are by far the most important. The $\left(\lambda_{0} \mu_{0}\right)=(22)$ tensors are also significant. In a restricted $Q_{1}=Q_{2}=4,(\lambda \mu)=(04)$ basis these would give rise to a nearly pure-rotational spectrum for the $0^{+}, 2^{+}, 4^{+}$excitations. The $\left(\lambda_{0} \mu_{0}\right)=(44)$ tensor, which in this restricted basis might have been responsible for large deviations from a pure $L(L+1)$

TABLE 2

The SU(3) reduced matrix elements $\left\langle\bar{Q}_{1} \bar{Q}_{2}(\bar{\lambda} \bar{\mu})\left\|\mathscr{X}^{\left(\lambda_{0} \mu_{0}\right)}\right\| Q_{1} Q_{2}(\lambda \mu)\right\rangle_{\rho_{0}}=\left\langle 44(04) H \mathscr{X}^{\left(\lambda_{0} \mu_{0}\right)} H \mid 44(04)\right\rangle$

\begin{tabular}{rrcccc}
\hline$\left(\lambda_{0} \mu_{0}\right)$ & Central pot. & Kin. energy & Coulomb pot. & Total energy \\
\hline$(00)$ & -799.94 & 508.46 & 26.99 & $\ddots$ & -264.51 \\
$(22)$ & 51.89 & -29.54 & -1.49 & & 20.86 \\
$(44)$ & -0.49 & 0.069 & -0.007 & & -0.428 \\
\hline
\end{tabular}

All numbers are given in MeV. 
excitation spectrum, is seen to be much less significant. It is also interesting to note how the reduced matrix elements are made up from the various $\bar{q}_{i}, q_{i},\left(\lambda_{\rho} \mu_{\rho}\right), \ldots$ components listed in table 1. By far the largest contributions come from the terms with $q_{1}+q_{2}=0$. The contributions from the $q_{1}+q_{2}=2$ terms are only $16.5 \%$ $15.1 \%$, and $14.5 \%$ of the former for the central potential, kinetic energy, and Coulomb potential, respectively. The corresponding numbers for the $q_{1}+q_{2}=4$ terms are $0.25 \%, 0.06 \%$, and $0.11 \%$; and the contributions from terms with $q_{1}+q_{2}=6$ or 8 are completely negligible. Since terms with $\bar{q}_{1}+\bar{q}_{2}, q_{1}+q_{2}>0$ arise from the expansion of the $\exp \{\rho\}, \exp \{\tau\}$ factors of the B-S transform, it can be seen that only the lowest terms in these expansions make significant contributions to the reduced matrix elements. This may lead to possible simplifications in a basis in which larger values of $Q_{1}, Q_{2}$ are included.

By combining the reduced matrix elements of table 2 with the appropriate $\mathrm{SU}(3) \supset \mathrm{R}(3)$ Wigner coefficients a total binding energy of $-78.73 \mathrm{MeV}$ is obtained for the ${ }^{12} \mathrm{C}$ nucleus, compared with $-78.56 \mathrm{MeV}$ of an earlier calculation ${ }^{11}$ ) based on the same Hamiltonian.

\section{The four-cluster system}

For the simple three-cluster system, with only two relative motion functions, much of the SU(3) technology collapses to simple SU(2) technology since many of the needed recoupling coefficients contain $\mathrm{U}(3)$ representations with at most tworowed tableaux. It may therefore be useful to study a cluster system made up of four fragments, each with 0 s oscillator functions in its internal degrees of freedom. This system will demonstrate the full complexity of the new method. The generalization to multicluster systems is then straightforward.

For a four-cluster system, with three relative motion functions, the function, $A$, which generates the BS transform is expanded by

$$
\begin{aligned}
\prod_{i=1}^{3} A\left(\boldsymbol{K}_{i}, \boldsymbol{R}_{i}\right) & =\sum_{Q_{1} Q_{2} Q_{3}} \sum_{\left(\lambda_{12} \mu_{12}\right)} \sum_{(\lambda \mu) \alpha} \\
& \times\left[\left[P\left(K_{1}\right)^{\left(Q_{1} 0\right)} \times P\left(K_{2}\right)^{\left(Q_{2} 0\right)}\right]^{\left(\lambda_{12} \mu_{12}\right)} \times P\left(K_{3}\right)^{\left(Q_{3} 0\right)}\right]_{x}^{(\lambda \mu)} \\
& \times\left[\left[\chi\left(R_{1}\right)^{\left(Q_{1} 0\right)} \times \chi\left(R_{2}\right)^{\left(Q_{2} 0\right)}\right]^{\left(\lambda_{12} \mu_{12}\right)} \times \chi\left(R_{3}\right)^{\left(Q_{3} 0\right)}\right]_{x}^{(\lambda \mu)^{*}},
\end{aligned}
$$

and, with $\bar{R}=\bar{R}_{1}, \bar{R}_{2}, \overline{\boldsymbol{R}}_{3}$, etc., the BS transform of a kernel $\mathscr{K}(\overline{\boldsymbol{R}}, \boldsymbol{R})$ is expanded in terms of the basic $S U(\overrightarrow{3})$ coupled $K$-space irreducible tensors by

$$
\begin{aligned}
H(\overline{\boldsymbol{K}}, \boldsymbol{K}) & =\sum_{\bar{Q}_{1} \bar{Q}_{2} \bar{Q}_{3}\left(\overline{\bar{\lambda}}_{12} \bar{\mu}_{12}\right)(\bar{\lambda} \bar{\mu})} \sum_{Q_{1} Q_{2} Q_{3}\left(\lambda_{12} \mu_{12}\right)(\lambda \mu)} \sum_{\left(\lambda_{0} \mu_{0}\right) \rho_{0}} \\
& \times\left\langle\left(\bar{Q}_{1} \bar{Q}_{2}\right)\left(\bar{\lambda}_{12} \bar{\mu}_{12}\right) \bar{Q}_{3}(\overline{\lambda \mu})\left\|\mathscr{K}^{\left(\lambda_{0} \mu_{0}\right)}\right\| Q_{1} Q_{2}\left(\lambda_{12} \mu_{12}\right) Q_{3}(\lambda \mu)\right\rangle_{\rho_{0}}
\end{aligned}
$$




$$
\begin{aligned}
& \times\left[\left[\left[P\left(\overline{\boldsymbol{K}}_{1}\right)^{\left(\bar{Q}_{1} 0\right)} \times P\left(\overline{\boldsymbol{K}}_{2}\right)^{\left(\bar{Q}_{2} 0\right)}\right]^{\left(\bar{\lambda}_{12} \bar{\mu}_{12}\right)} \times P\left(\overline{\boldsymbol{K}}_{3}\right)^{\left(\bar{Q}_{3} 0\right)}\right]^{(\bar{\lambda} \bar{\mu})}\right. \\
& \left.\times\left[\left[P\left(\boldsymbol{K}_{1}^{*}\right)^{\left(0 Q_{1}\right)} \times P\left(K_{2}^{*}\right)^{\left(0 Q_{2}\right)}\right]^{\left(\mu_{12} \lambda_{12}\right)} \times P\left(\boldsymbol{K}_{3}^{*}\right)^{\left(0 Q_{3}\right)}\right]^{(\mu \lambda)}\right]_{L_{0}=0}^{\left(\lambda_{0} \mu_{0}\right) \rho_{0}} .
\end{aligned}
$$

An expansion of the BS transform in terms of the basic $\boldsymbol{K}$-space tensors again leads directly to the needed SU(3) reduced matrix elements. The function $H(\vec{K}, \boldsymbol{K})$ can again be expanded in terms of Gaussians, $\exp \{\rho\} \exp \{\sigma\} \exp \{\tau\}$, [see eqs. (18), (19)]. The expansion of each factor is accomplished in analogy with the earlier simpler case. The $\sigma$-factor gives

$$
\begin{aligned}
\exp \{ & \left.\sum_{i=j=1}^{3} \sigma_{i j}\left(\overline{\boldsymbol{K}}_{i} \cdot \boldsymbol{K}_{j}^{*}\right)\right\} \\
= & \sum C_{\sigma}\left(l_{1}+m_{1}+n_{1}, l_{2}+m_{2}+n_{2}, l_{3}+m_{3}+n_{3}, l_{1}+l_{2}+l_{3},\right. \\
& \left.m_{1}+m_{2}+m_{3}, n_{1}+n_{2}+n_{3} ;\left(\lambda_{12}^{\prime} \mu_{12}^{\prime}\right),\left(\hat{\lambda}_{12}^{\prime \prime} \mu_{12}^{\prime \prime}\right),\left(\lambda_{\sigma} \mu_{\sigma}\right)\right) \\
& \times\left[\left[\left[P\left(\overline{\boldsymbol{K}}_{1}\right)^{\left(l_{1}+m_{1}+n_{1}, 0\right)} \times P\left(\overline{\boldsymbol{K}}_{2}\right)^{\left(l_{2}+m_{2}+n_{2}, 0\right)}\right]^{\left(\lambda_{12}^{\prime} \mu_{12}^{\prime}\right)} \times P\left(\overline{\boldsymbol{K}}_{3}\right)^{\left(l_{3}+m_{3}+n_{3}, 0\right)}\right]^{\left(\lambda_{\sigma} \mu_{\sigma}\right)}\right. \\
& \times\left[\left[P\left(\boldsymbol{K}_{1}^{*}\right)^{\left(0, l_{1}+l_{2}+l_{3}\right)} \times P\left(\boldsymbol{K}_{2}^{*}\right)^{\left(0, m_{1}+m_{2}+m_{3}\right)}\right]^{\left(\mu_{12}^{\prime \prime} \lambda_{12}^{\prime \prime}\right)}\right. \\
& \left.\left.\times P\left(\boldsymbol{K}_{3}^{*}\right)^{\left(0, n_{1}+n_{2}+n_{3}\right)}\right]^{\left(\mu_{\sigma} \bar{\lambda}_{\sigma}\right)}\right]_{0}^{(00)},
\end{aligned}
$$

where

$$
\begin{aligned}
& C_{\sigma}=\sum_{\substack{l_{i} m_{i} n_{i} \\
i=1.2,3}} \sigma_{11}^{l_{1}} \sigma_{12}^{m_{1}} \sigma_{13}^{n_{1}} \sigma_{21}^{l_{2}} \sigma_{22}^{m_{2}} \sigma_{23}^{n_{2}} \sigma_{31}^{l_{3}} \sigma_{32}^{m_{3}} \sigma_{33}^{n_{3}} \\
& \times\left[\operatorname{dim}\left(\lambda_{\sigma} \mu_{\sigma}\right)\right]^{\frac{1}{3}}\left[\left(l_{1}+m_{1}+n_{1}\right) !\left(l_{2}+m_{2}+n_{2}\right) !\left(l_{3}+m_{3}+n_{3}\right) !\right. \\
& \left.\times\left(l_{1}+l_{2}+l_{3}\right) !\left(m_{1}+m_{2}+m_{3}\right) !\left(n_{1}+n_{2}+n_{3}\right) !\right]^{\frac{1}{3}} / \\
& \times\left[l_{1} ! l_{2} ! l_{3} ! m_{1} ! m_{2} ! m_{3} ! n_{1} ! n_{2} ! n_{3} !\right] \\
& \times \sum_{\left(\lambda_{12}^{\prime} \mu_{12}^{\prime \prime}\right)}\left[\begin{array}{ccc}
\left(l_{1} 0\right) & \left(m_{1} 0\right) & \left(l_{1}+m_{1}, 0\right) \\
\left(l_{2} 0\right) & \left(m_{2} 0\right) & \left(l_{2}+m_{2}, 0\right) \\
\left(l_{1}+l_{2}, 0\right) & \left(m_{1}+m_{2}, 0\right) & \left(\lambda_{12}^{\prime \prime \prime} \mu_{12}^{\prime \prime}\right)
\end{array}\right] \\
& \times\left\{\begin{array}{ccc}
\left(l_{1}+l_{2}, 0\right) & \left(m_{1}+m_{2}, 0\right) & \left(\lambda_{12}^{\prime \prime \prime} \mu_{12}^{\prime \prime \prime}\right) \\
\left(l_{3} 0\right) & \left(m_{3} 0\right) & \left(l_{3}+m_{3}, 0\right) \\
\left(l_{1}+l_{2}+l_{3}, 0\right) & \left(m_{1}+m_{2}+m_{3}, 0\right) & \left(\lambda_{12}^{\prime \prime} \mu_{12}^{\prime \prime}\right)
\end{array}\right] \\
& \times\left[\begin{array}{ccc}
\left(\lambda_{12}^{\prime \prime \prime} \mu_{12}^{\prime \prime \prime}\right) & \left(n_{1}+n_{2}, 0\right) & \left(\lambda_{12}^{\prime} \mu_{12}^{\prime}\right) \\
\left(l_{3}+m_{3}, 0\right) & \left(n_{3}, 0\right) & \left(l_{3}+m_{3}+n_{3}, 0\right) \\
\left(\lambda_{12}^{\prime \prime} \mu_{12}^{\prime \prime}\right) & \left(n_{1}+n_{2}+n_{3}, 0\right) & \left(\lambda_{\sigma} \mu_{\sigma}\right)
\end{array}\right]
\end{aligned}
$$




$$
\times\left[\begin{array}{ccc}
\left(l_{1}+m_{1}, 0\right) & \left(n_{1}, 0\right) & \left(l_{1}+m_{1}+n_{1}, 0\right) \\
\left(l_{2}+m_{2}, 0\right) & \left(n_{2}, 0\right) & \left(l_{2}+m_{2}+n_{2}, 0\right) \\
\left(\lambda_{12}^{\prime \prime \prime} \mu_{12}^{\prime \prime \prime}\right) & \left(n_{1}+n_{2}, 0\right) & \left(\lambda_{12}^{\prime} \mu_{12}^{\prime}\right)
\end{array}\right]
$$

The expansion of the $\rho$ (and similarly the $\tau$ ) factor is given by

$$
\begin{aligned}
\exp \{ & \left.\sum_{i, j=1}^{3} \rho_{i j}\left(\overline{\boldsymbol{K}}_{i} \cdot \bar{K}_{j}\right)\right\}=\sum_{\bar{q}_{1} \bar{q}_{2} \bar{q}_{3}} \sum_{\left(\lambda_{\rho_{12}} \mu_{\rho_{12}}\right)} \sum_{\left(\lambda_{\rho} \mu_{\rho}\right)} \\
& \times B_{\rho}\left(\bar{q}_{1}, \bar{q}_{2}, \bar{q}_{3} ;\left(\lambda_{\rho_{12}} \mu_{\rho_{12}}\right),\left(\lambda_{\rho} \mu_{\rho}\right)\right) \\
& \times\left[\left[P\left(\boldsymbol{K}_{1}\right)^{\left(\bar{q}_{1} 0\right)} \times P\left(\bar{K}_{2}\right)^{\left(\bar{q}_{2} 0\right)}\right]^{\left(\lambda_{\rho_{12}} \mu_{\rho_{12}}\right)} \times P\left(\bar{K}_{3}\right)^{\left(\bar{q}_{3} 0\right)}\right]_{L_{\rho}=0}^{\left(\lambda_{\rho} \mu_{\rho}\right)},
\end{aligned}
$$

where

$$
\begin{aligned}
& B_{\rho}=\sum_{l, m, n} \rho_{a a}^{l} \rho_{b b}^{m} \rho_{c c}^{n} \frac{[(2 l+1) !(2 m+1) !(2 n+1) !]^{\frac{1}{2}}}{l ! m ! n !} \\
& \times \sum_{\left(\lambda_{a b} \mu_{a b}\right)}\left\langle(2 l, 0) 0 ;(2 m, 0) 0 \|\left(\lambda_{a b} \mu_{a b}\right) 0\right\rangle \\
& \times\left\langle\left(\lambda_{a b} \mu_{a b}\right) 0 ;(2 n, 0) 0 \|\left(\lambda_{\rho} \mu_{\rho}\right) 0\right\rangle \\
& \times d_{2 l, 2 m,\left(\lambda_{a b} \mu_{a b}\right), 2 n ;}^{\left(\lambda_{\rho} \mu_{\rho}\right)} \bar{q}_{1, \bar{q}_{2,}\left(\lambda_{\rho_{12}} \mu_{\rho_{12}}\right), \bar{q}_{3}}\left(\beta_{1}, \beta_{2}, \beta_{3}\right) .
\end{aligned}
$$

The symmetric quadratic form has again first been put into diagonal form,

$$
\sum_{i, j=1}^{3} \rho_{i j}\left(\boldsymbol{K}_{i} \cdot \overline{\boldsymbol{K}}_{j}\right)=\rho_{a a}\left(\boldsymbol{K}_{a} \cdot \overline{\boldsymbol{K}}_{a}\right)+\rho_{b b}\left(\overline{\boldsymbol{K}}_{b} \cdot \overline{\boldsymbol{K}}_{b}\right)+\rho_{c c}\left(\overline{\boldsymbol{K}}_{c} \cdot \boldsymbol{K}_{c}\right)
$$

by the transformation

$$
\bar{K}^{\prime}=O \bar{K}=O_{3} O_{2} O_{1} \bar{K}
$$

with $\boldsymbol{R}^{\prime}=\left(\boldsymbol{K}_{a}, \boldsymbol{R}_{b}, \mathbb{R}_{c}\right), \boldsymbol{K}=\left(\boldsymbol{R}_{1}, \boldsymbol{K}_{2}, \boldsymbol{R}_{3}\right) ;$ and with

$$
O=\left(\begin{array}{ccc}
c_{3} & -s_{3} & 0 \\
s_{3} & c_{3} & 0 \\
0 & 0 & 1
\end{array}\right)\left(\begin{array}{ccc}
c_{2} & 0 & -s_{2} \\
0 & 1 & 0 \\
s_{2} & 0 & c_{2}
\end{array}\right)\left(\begin{array}{ccc}
c_{1} & -s_{1} & 0 \\
s_{1} & c_{1} & 0 \\
0 & 0 & 1
\end{array}\right),
$$

and $c_{i}=\cos \frac{1}{2} \beta_{i}$ and $s_{i}=\sin \frac{1}{2} \beta_{i}$. The generalized Moshinsky bracket which takes us from the $\mathrm{SU}(3)$ coupled $\left[[(2 l, 0) \times(2 m, 0)]\left(\lambda_{a b} \mu_{a b}\right) \times(2 n, 0)\right]\left(\lambda_{\rho} \mu_{\rho}\right)$ scheme in $\boldsymbol{K}^{\prime}$ 
space to the $\left[\left[\left(\bar{q}_{1} 0\right) \times\left(\bar{q}_{2} 0\right)\right]\left(\lambda_{\rho_{12}} \mu_{\rho_{12}}\right) \times\left(\bar{q}_{3} 0\right)\right]\left(\lambda_{\rho} \mu_{\rho}\right)$ scheme in $\bar{K}$ space is now given by a special $\mathrm{U}(3) d$-function ${ }^{18,19}$ )

$$
\begin{aligned}
& d_{2 l, 2 m,\left(\lambda_{a b} \mu_{a b}\right), 2 n ; \bar{q}_{1}, \bar{q}_{2,}\left(\lambda_{\rho_{12}} \mu_{\rho_{12}}\right), \bar{q}_{3}}\left(\beta_{1}, \beta_{2}, \beta_{3}\right)
\end{aligned}
$$

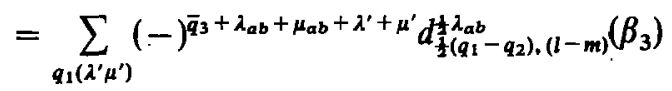

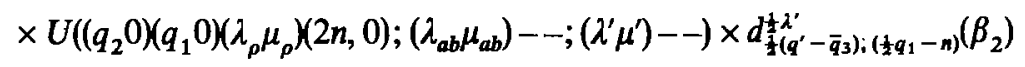

$$
\begin{aligned}
& \times U\left(\left(q_{2} 0\right)\left(q^{\prime} 0\right)\left(\lambda_{\rho} \mu_{\rho}\right)\left(\bar{q}_{3} 0\right) ;\left(\lambda_{\rho_{12}} \mu_{\rho_{12}}\right)--;\left(\lambda^{\prime} \mu^{\prime}\right)--\right) \times d d_{f\left(\bar{q}_{1}-\bar{q}_{2}\right), t\left(q^{\prime}-q_{2}\right)}^{\lambda_{\rho_{12}}}\left(\beta_{1}\right),
\end{aligned}
$$

with $q_{1}, q_{2}, q^{\prime}$; restricted by $q_{1}+q_{2}=2 l+2 m ; q^{\prime}+\bar{q}_{3}=q_{1}+2 n ; q^{\prime}+q_{2}=\bar{q}_{1}+\bar{q}_{2}$. This is a slight generalization of the formula given by Chacon ${ }^{19}$ ) for the SU(3) $d$-function. This generalization is necessitated by the fact that the Young tableau for $\left(\lambda_{\rho} \mu_{\rho}\right)$ built from $2 l+2 m+2 n=\bar{q}_{1}+\bar{q}_{2}+\bar{q}_{3}$ oscillator quanta will in general be three-rowed, so that the two recoupling coefficients must be bona fide SU(3) Racah coefficients.

The $\rho, \sigma$ and $\tau$ factors are finally assembled in $\mathrm{SU}(3)$ coupled tensor form by a straightforward generalization of eq. (37) with the $C_{\sigma}, B_{\rho}$, and $B_{\tau}$ of appropriate quantum numbers (e.g. $C_{\sigma} \equiv C_{\sigma}\left(\bar{Q}_{1}-\bar{q}_{1}, \bar{Q}_{2}-\bar{q}_{2}, \bar{Q}_{3}-\bar{q}_{3}, Q_{1}-q_{1}, Q_{2}-q_{2}, Q_{3}-q_{3}\right.$; $\left.\left(\lambda_{12}^{\prime} \mu_{12}^{\prime}\right),\left(\lambda_{12}^{\prime \prime} \mu_{12}^{\prime \prime}\right),\left(\lambda_{\sigma} \mu_{\sigma}\right)\right)$; and with a $Z$-function which is now generalized to

$$
\begin{aligned}
& Z=\sum_{\rho^{\prime} \rho^{\prime \prime \prime}} U\left(\left(\lambda_{\rho} \mu_{\rho}\right)\left(\lambda_{\sigma} \mu_{\sigma}\right)\left(\lambda_{\rho} \mu_{\rho}\right)\left(\mu_{\sigma} \lambda_{\sigma}\right) ;(\overline{\lambda \mu}) \rho^{\prime} \rho^{\prime} ;(00)--\right) \\
& \times\left\{\sum_{\bar{\rho}_{0}}\left\langle\left(\lambda_{\rho} \mu_{\rho}\right) L_{\rho}=0 ;\left(\mu_{\tau} \lambda_{\tau}\right) L_{\tau}=0 \|\left(\lambda_{0} \mu_{0}\right) L_{0}=0\right\rangle_{\bar{\rho}_{0}}\right. \\
& \left.\times U\left((\overline{\lambda \mu})\left(\mu_{\sigma} \lambda_{\sigma}\right)\left(\lambda_{0} \mu_{0}\right)\left(\mu_{\tau} \lambda_{\tau}\right) ;\left(\lambda_{\rho} \mu_{\rho}\right) \rho^{\prime} \bar{\rho}_{0} ;(\mu \lambda) \rho^{\prime \prime} \rho_{0}\right)\right\} \\
& \times\left[\prod_{i=1}^{3}\left(\begin{array}{l}
\bar{Q}_{i} \\
\bar{q}_{i}
\end{array}\right)\left(\begin{array}{l}
Q_{i} \\
q_{i}
\end{array}\right)\right]^{\frac{1}{2}} \\
& \times\left[\begin{array}{ccc}
\left(\bar{q}_{1} 0\right) & \left(\bar{q}_{2} 0\right) & \left(\lambda_{\rho_{12}} \mu_{\rho_{12}}\right) \\
\left(\bar{Q}_{1}-\bar{q}_{1}, 0\right) & \left(\bar{Q}_{2}-\bar{q}_{2}, 0\right) & \left(\lambda_{12}^{\prime} \mu_{12}^{\prime}\right) \\
\left(\bar{Q}_{1} 0\right) & \left(\bar{Q}_{2} 0\right) & \left(\bar{\lambda}_{12} \bar{\mu}_{12}\right)
\end{array}\right] \\
& \times\left[\begin{array}{cccc}
\left(\lambda_{\rho_{12}} \mu_{\rho_{12}}\right) & \left(\bar{q}_{3} 0\right) & \left(\lambda_{\rho} \mu_{\rho}\right) & - \\
\left(\lambda_{12}^{\prime} \mu_{12}^{\prime}\right) & \left(\bar{Q}_{3}-\bar{q}_{3}, 0\right) & \left(\lambda_{\sigma} \mu_{\sigma}\right) & - \\
\left(\lambda_{12} \bar{\mu}_{12}\right) & \left(\bar{Q}_{3} 0\right) & (\overline{\lambda \mu}) & - \\
- & - & . \rho^{\prime} & -
\end{array}\right] \\
& \times\left[\begin{array}{ccc}
\left(Q_{1}-q_{1}, 0\right) & \left(Q_{2}-q_{2}, 0\right) & \left(\lambda_{12}^{\prime \prime} \mu_{12}^{\prime \prime}\right) \\
\left(q_{1} 0\right) & \left(q_{2} 0\right) & \left(\lambda_{\tau_{12}} \mu_{\tau_{12}}\right) \\
\left(Q_{1} 0\right) & \left(Q_{2} 0\right) & \left(\lambda_{12} \mu_{12}\right)
\end{array}\right]
\end{aligned}
$$




$$
\times\left(\begin{array}{cccc}
\left(\lambda_{12}^{\prime \prime} \mu_{12}^{\prime \prime}\right) & \left(Q_{3}-q_{3}, 0\right) & \left(\lambda_{\sigma} \mu_{\sigma}\right) & - \\
\left(\lambda_{\tau_{12}} \mu_{\tau_{12}}\right) & \left(q_{3} 0\right) & \left(\lambda_{\tau} \mu_{\tau}\right) & - \\
\left(\lambda_{12} \mu_{12}\right) & \left(Q_{3} 0\right) & (\lambda \mu) & - \\
- & - & \rho^{\prime \prime}
\end{array}\right)
$$

Both the $U$-coefficients and two of the $9(\lambda \mu)$ recoupling coefficients are now bona fide SU(3) coefficients including some representations with three-rowed tableaux. Even in these two $9(\lambda \mu)$ recoupling coefficients five of the six $S U(3)$ couplings are automatically free of outer multiplicities. Unnecessary multiplicity labels are replaced by a dash.

The generalization to heavier multi-cluster systems is now straightforward. Cluster systems whith one or more heavy fragments can also be included by limiting the excitations in some of the relative motion functions in the $n$-cluster system to their lowest Pauli-allowed values. In an aggregate made up of $m$ 0s clusters $(m<n)$, with $m-1$ relative motion functions of equal oscillator frequency, and with oscillator quanta $Q_{1}, Q_{2}, \ldots Q_{m-1}$ frozen into their lowest Pauli-allowed values, the antisymmetrizer assures that the wave function of such an aggregate is itself the internal wave function of a heavy-cluster fragment. In our coupling scheme such a wave function corresponds to a ground-state shell-model configuration of definite $\mathrm{SU}(3)$ symmetry $\left(\lambda_{\mathrm{c}} \mu_{\mathrm{c}}\right)$ of the heavy-fragment nucleus. The BS transform of such a heavyfragment internal wave function can simply be denoted by $\left.P\left(\boldsymbol{K}_{\mathrm{int}}, \ldots, \boldsymbol{K}_{\mathrm{int}}\right)_{m-1}\right)_{\alpha_{c}}^{\left(\lambda_{c} \mu_{c}\right)}$ for heavy fragments with $5 \leqq A \leqq 16$. The antisymmetrizer assures that the order and the details of the SU(3) coupling in the $K_{1}, \ldots, K_{m-1}$ subspace are immaterial; and the heavy-cluster fragment can be characterized by $\left(\lambda_{\mathrm{c}} \mu_{\mathrm{c}}\right), \alpha_{\mathrm{c}}$ alone.

In the technique outlined so far the details of antisymmetrization are handled by an expansion in terms of double coset generators. For an $n$-cluster problem, with large $n$, the number of terms in such an expansion may become prohibitively large, and more indirect methods may be preferable. Such methods have been used to advantage for the norm problem of a series of nuclei with $12 \leqq A \leqq 24$, described in a (heavy-fragment $+\alpha$-particle) cluster model basis ${ }^{7}$ ). The BS transform of the full $n$-cluster problem is of simple Gaussian form, $\sum_{\beta} a_{\beta} \exp \{\rho(\beta)\} \exp \{\sigma(\beta)\} \exp \{\tau(\beta)\}$, with a $\beta$-sum which runs over a large number of double coset generators. For the restricted cluster problem, e.g. for a single heavy fragment with one or two additional Os cluster components, this sum is reduced to one with a manageable number of terms. However, the BS transform is no longer of simple Gaussian form. In this case the general form of the BS transform of the restricted cluster problem can be established from the full Gaussian form for the unrestricted $n$-cluster problem by expanding the exponentials containing internal variables and limiting the powers of $\overline{\boldsymbol{K}}_{\text {int } 1_{1}}, \ldots, \overline{\boldsymbol{K}}_{\text {int }_{m-1}}, \boldsymbol{K}_{\text {int }_{1}}^{*}, \ldots, \boldsymbol{K}_{\text {int }_{m-1}}^{*}$ to their minimum Pauli-allowed values, as dictated by the internal wave function of the heavy fragment. In a (heavy-fragment $+\alpha+\alpha$ ) three-cluster system, e.g., the two relative motion vectors can be denoted by $\boldsymbol{R}_{1}, \boldsymbol{R}_{\mathbf{2}}$ with BS transforms $K_{1}, K_{2}$. The exponential factors made 'up from $\boldsymbol{K}_{1}, \boldsymbol{K}_{2}, K_{1}^{*}, K_{2}^{*}$ 
variables only may be handled by the techniques of sect. 2 . The expansion of exponentials with cross terms between the $\overline{\boldsymbol{K}}_{\mathrm{int}}, \boldsymbol{K}_{1}^{*}, \boldsymbol{K}_{2}^{*}$, and $\boldsymbol{K}_{\mathrm{int}}^{*}, \boldsymbol{K}_{1}, \overline{\boldsymbol{K}}_{2}$ can be combined with the expansion of the exponentials of pure $\overline{\boldsymbol{K}}_{\text {int }}, \boldsymbol{K}_{\text {int }}^{*}$ terms to yield $\boldsymbol{K}$-space polynomials of the type

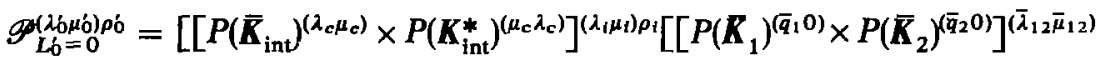

$$
\begin{aligned}
& \left.\left.\times\left[P\left(K_{1}^{*}\right)^{\left(0 q_{1}\right)} \times P\left(K_{2}^{*}\right)^{\left(0 q_{2}\right)}\right]^{\left(\mu_{12} \lambda_{12}\right)}\right]^{\left(\Lambda_{12} M_{12}\right) \rho_{12}}\right]_{L_{0}=0}^{\left(\lambda 6 \mu \mu_{0}\right) \rho_{6}}
\end{aligned}
$$

The full B-S transform is then assembled by combining these polynomials with the $\bar{K}_{1}, \mathbb{K}_{2}, \boldsymbol{K}_{1}^{*}, \boldsymbol{K}_{2}^{*}$ space exponentials, and as yet undetermined coefficients. If the heavy fragment is a p-shell nucleus in a state of highest possible $\mathrm{SU}(3)$ symmetry $\left(\lambda_{\mathrm{c}} \mu_{\mathrm{c}}\right)$; the number of possible combinations $\left(\bar{\lambda}_{12} \bar{\mu}_{12}\right),\left(\lambda_{12} \mu_{12}\right),\left(\Lambda_{12} M_{12}\right) \rho_{12},\left(\lambda_{i} \mu_{i}\right) \rho_{i}$, is severely limited by SU(3) coupling rules and the fact that the $\bar{q}_{1}, \bar{q}_{2}, q_{1}, q_{2}$ are restricted to very small numbers. The total number of undetermined coefficients is thus a manageable number, and these may be evaluated by indirect methods of the type used with considerable success ${ }^{7}$ ) for the simpler (heavy-fragment $+\alpha$-particle) cluster system.

Detailed microscopic treatments of nuclear cluster problems by resonating group methods have until now been limited to very light nuclei or cluster systems made up of closed-shell nuclei. By using a cluster-model basis in which the relative motion and internal oscillator wave functions are given in SU(3) coupled form, it has been possible to combine the power of integral transform methods with SU(3) recoupling techniques to derive closed expressions for matrix elements of more challenging cluster problems. Multi-cluster systems can be handled as easily as the simple twocluster system. The SU(3) reduced matrix elements are evaluated by integral transform methods and are expressed mainly in terms of SU(3) recoupling coefficients. Matrix elements in a basis of good angular momentum are then constructed trivially by combining these $\mathrm{SU}(3)$ reduced matrix elements with readily available $\mathrm{SU}(3)$ Wigner coefficients.

\section{References}

1) H. H. Hackenbroich, Symp. on present status and novel developments in the nuclear many-body problem, Rome (Editrice Compositori, Bologna, 1973)

2) H. Stöwe and W. Zahn, Nucl. Phys. A289 (1977) 317; Z. Phys. A286 (1978) 173

3) P. Kramer, M. Moshinsky and T. H. Seligman, in Group theory and its applications, vol. 3, ed. E. M. Loebl (Academic Press, New York, 1975), and references therein

4) T. H. Seligman and W. Zahn, J. of Phys. G2 (1976) 79;

K. T. Hecht, T. H. Seligman and W. Zahn, to be published

5) W. Zahn, Burg monographs in science, vol. 2 (Burg Verlag, Basel, 1975);

H. H. Hackenbroich, T. H. Seligman and W. Zahn, Helv. Phys. Acta 50 (1977) 723

6) T. H. Seligman and W. Zahn, Helv. Phys. Acta 49 (1976) 217

7) K. T. Hecht and W. Zahn, Nuç. Phys., in press

8) J. P. Draayer and Y. Akiyama, J. Math. Phys. 14 (1973) 1904; Comp. Phys. Commun. 5 (1973) 405 
9) P. Kramer and T. H. Seligman, Nucl. Phys. A136 (1969) 545; A186 (1972) 49;

A. Antillon and T. H. Seligman, to be published

10) H. Eikemeier and H. H. Hackenbroich, Z. Phys. 195 (1966) 412; Nucl. Phys. A169 (1971) 407

11) H. Hutzelmeyer and H. H. Hackenbroich, Z. Phys. 232 (1970) 356

12) J. P. Elliott, Proc. Roy. Soc. A245 (1958) 128, 562

13) P. Kramer and T. H. Seligman, Z. Phys. 219 (1969) 105

14) R. T. Sharp, H. C. von Baeyer and S. C. Pieper, Nucl. Phys. A127 (1969) 513

15) A. R. Edmonds, Angular momentum in quantum mechanics (Princeton University Press, 1957)

16) T. A. Brody and M. Moshinsky, Rev. Mex. Fiz. 9 (1960) 181

17) B. Kaufmann and C. Noack, J. Math. Phys. 6 (1965) 142

18) E. Chacon and M. Moshinsky, Phys. Lett. 23 (1966) 567

19) E. Chacon, Rev. Mex. Fis. 17 (1968) 315 